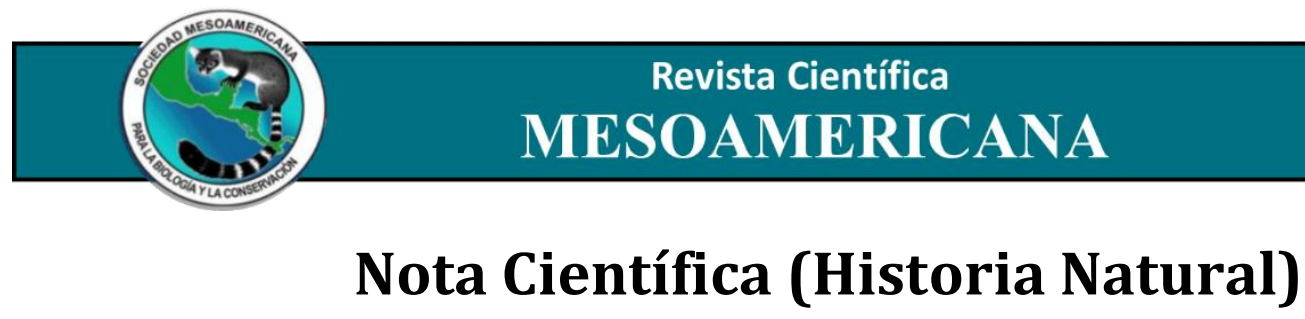

\title{
Observaciones biológicas de Hyphilaria thasus (Stoll) (Lepidoptera, Riodinidae) y su parasitoide en Panamá
}

\author{
Alonso Santos Murgas ${ }^{1}$ (iD \& Luis A. Jaen ${ }^{2}$ \\ Universidad de Panamá, Facultad de Ciencias Naturales Exactas y Tecnología, ${ }^{1}$ Departamento de Zoología. \\ ${ }^{2}$ Departamento de Genética y Biología Molecular. ${ }^{1,2}$ Sociedad Mesoamericana para la Biología y la Conservación- \\ Capitulo de Panamá. Email: santosmurgasa@gmail.com ; luisangeljaen@gmail.com
}

Fecha de Recepción: 29-I-2020. Fecha de Aceptación: 15-VI-2020. Fecha de Publicación: 25-VII-2020.

\section{RESUMEN}

Se observaron cinco larvas de Hyphilaria thasus (Stoll, 1780) del tercer y último estadio, alimentándose de las hojas de un híbrido de la orquídea Dendrobium sp, ubicados en las Villas de Arraiján, Juan Demóstenes Arosemena, Arraiján, provincia de Panamá Oeste, República de Panamá. Se extrajeron las cinco larvas de la orquídea Dendrobium sp. del sitio donde se encontraban y se llevaron al laboratorio a temperatura ambiente, se colocaron en una jaula de malla de tela fina, con hojas de la planta para que continuaran alimentándose y siguiera su desarrollo. Luego de pasar tres días puparon las 5 larvas, posteriormente al pasar 3 días, salió de una de las pupas de la mariposa de $H$. thasus, una larva de mosca que pupó enseguida metiéndose sobre la cama de arena que colocamos dentro de la jaula en un petri de cristal; la mosca parasitoide corresponde a Lespesia archippivora (Riley) (Diptera: Tachinidae) que eclosionó a los 17 día de salir de la pupa de H. thasus. Cuatro adultos de la mariposa $H$. thasus lograron salir al cabo de 16 días después de pupar.

Palabras Clave: Hospedero, Diptera, parasitoides, Dendrobium, orquídeas.

\section{Biological observations of the Hyphilaria thasus (Stoll) (Lepidoptera, Riodinidae) and parasitoid in Panama}

\begin{abstract}
We observed five larvae of Hyphilaria Thasus in their third and last stage, feeding on the leaves of a hybrid of the orchid Dendrobium sp. The larvae were found in the Villas of Arraijan, West Panama. The researcher extracted the five larvae from the orchid Dendrobium sp. and took them to the laboratory. They were kept in a fine fabric mesh cage in room temperature and fed with the orchid's leaves to continue their development. After three days, the five larvae pupated. However, after three days, we noticed a fly larva of the parasitoid Lespesia archippivora (Riley) (Diptera: Tachinidae) emerged from $H$. thasus butterfly and hid immediately under the sand bed in the cage. The Lespesia archippivora (Riley) (Diptera: Tachinidae) fully developed after 17 days of leaving the body of the $H$. thasus butterfly. Also, four adults of the H. thasus butterfly emerged after 16 days from their pupa stage.
\end{abstract}

Keywords: Host, Diptera, parasitoid, Dendrobium, Orchids. 


\section{INTRODUCCIÓN}

La familia Riodinidae es una familia Pantropical de mariposas diurnas, pero la mayoría (93\%) de sus especies se encuentran en el Neotrópico (De Vries, 1997; Callaghan Lamas, 2004; Seraphin N., et al. 2018). El grupo muestra una notable diversidad de características morfológicas, ecológicas y conductuales; históricamente es la menos conocida de todas las mariposas diurnas de la superfamilia Papilionoidea (De Vries, 1997). Entre las características morfológicas más sobresalientes de los riodinidos, estas pequeñas mariposas muestran en sus alas líneas de colores muy llamativos, es un grupo muy diversos, en el campo generalmente pueden ser reconocidos por su hábito de posar bajo las hojas con las alas extendidas. Algo muy notable en campo de los riodinidos es que son muy activos desde el amanecer hasta el anochecer, algunas especies tienen periodos de vuelo restringido, es decir, algunos vuelan por la mañana, otros al medio día y otros antes del crepúsculo, se conoce que los adultos se alimentan de néctar de flores y las larvas de hojas de plantas y algunas de hojas muertas en el suelo del bosque, se pueden encontrar solitarias o en grupos, (http://janzen.bio.upenn.edu/caterpillars/database.lasso).

Las larvas de H. thasus se alimentan de plantas de la familia (Orchidaceae), viven en pequeños grupos alimentándose de las hojas de las orquídeas, donde pupan y llegan a la etapa adulta. Hyphilaria Hübner, (1819) es un género de la subfamilia Riodininae de la tribu Mesosemiini, el cual contiene cinco especies; H. nicia Hübner, (1819), H. anthias (Hewitson, 1874); H. anophthalma (Felder, 1865), H. parthenis (Westwood, 1851) y H. thasus (Stoll, 1780). La distribución actual de H. thasus va desde el sur este de México, Costa Rica, Panamá hasta Brazil y Paraguay. El objetivo de este trabajo es dar a conocer algunas observaciones biológicas de las larvas y enemigos naturales de H. thasus en Panamá.

\section{MATERIALES Y MÉTODOS}

Cinco larvas de la mariposa Hyphilaria thasus (Stoll, 1780) (Lepidoptera: Riiodinidae) fueron observadas en campo y posteriormente recolectadas como pupa en la República de Panamá, provincia de Panamá Oeste, ubicada en el corregimiento de Juan Demóstenes Arosemena, Distrito de Arraiján, en el jardín de una casa, dentro del Residencial Park Village; en las coordenadas, latitud 8 56 19.25 $\mathrm{N}$, longitud 79 44' 5.850 (Figura 1); 08.viii.2019, col. A. Santos M. Las cinco larvas se encontraban en hojas de un hibrido de orquídea Dendrobium sp. (Figuras 2, 3, 4). Estas se dejaron en la planta hasta que llegaran a pupar; luego fueron removidas de la planta donde se encontraban y colocadas dentro de platos petris sin tapa, dentro de una jaula rectangular de 12x12 pulgadas de malla fina, esperando la emergencia del adulto.

Para la identificación de la mariposa H. thasus se utilizó el libro de Kim Garwood y Richard Lehman. Butterflies of Central America, A Photophic Checklist of Common Species; Volumen 2: Lycaenidae y Riodinidae: The Hairstreaks and Metalmarks; 2012, Printed in McAllen, Texas, USA.

Para la mosca Tachinidae, Lespesia archippivora (Riley) se utilizó la revisión del género Lespesia de Guimarães (1983); Sabrosky (1980); Toma (2010) y también se comparó con los especímenes que existen en la Colección Nacional de Referencia de Insectos del Museo de Invertebrados G. B. Fairchild, de la Universidad de Panamá. Los especímenes criados se encuentran depositados en el Museo de Invertebrados G. B. Fairchild, Universidad de Panamá.

\section{RESULTADOS Y DISCUSIÓN}

Las larvas se dejaron en la planta hospedera hasta que puparan. Transcurrido tres días después de observar el tercer y último estadio de las larvas puparon. Ellas, al pupar se adhirieron a los mismos 
tallos y hojas de la orquídea (Figuras 3 y 4). Las pupas posteriormente fueron removidas y se colocaron en platos petris sin tapa, abiertos, dentro de una jaula de malla fina; al tercer día después de pupar, sale de una de las pupas de H. thasus, una larva de mosca; al observar el evento, esta pupa es traslada a otro plato petri con medio (1/2) centímetro de arena como sustrato, para que la larva de mosca pudiera enterrarse y pupar; transcurrido unas cinco horas la larva de mosca ya había formado su capullo y pupado cerca de la carcasa de la pupa de $H$. thasus, sobre el sustrato de arena, introduciéndose hasta el fondo del Petri, justo debajo de la carcasa de la pupa de H. thasus.

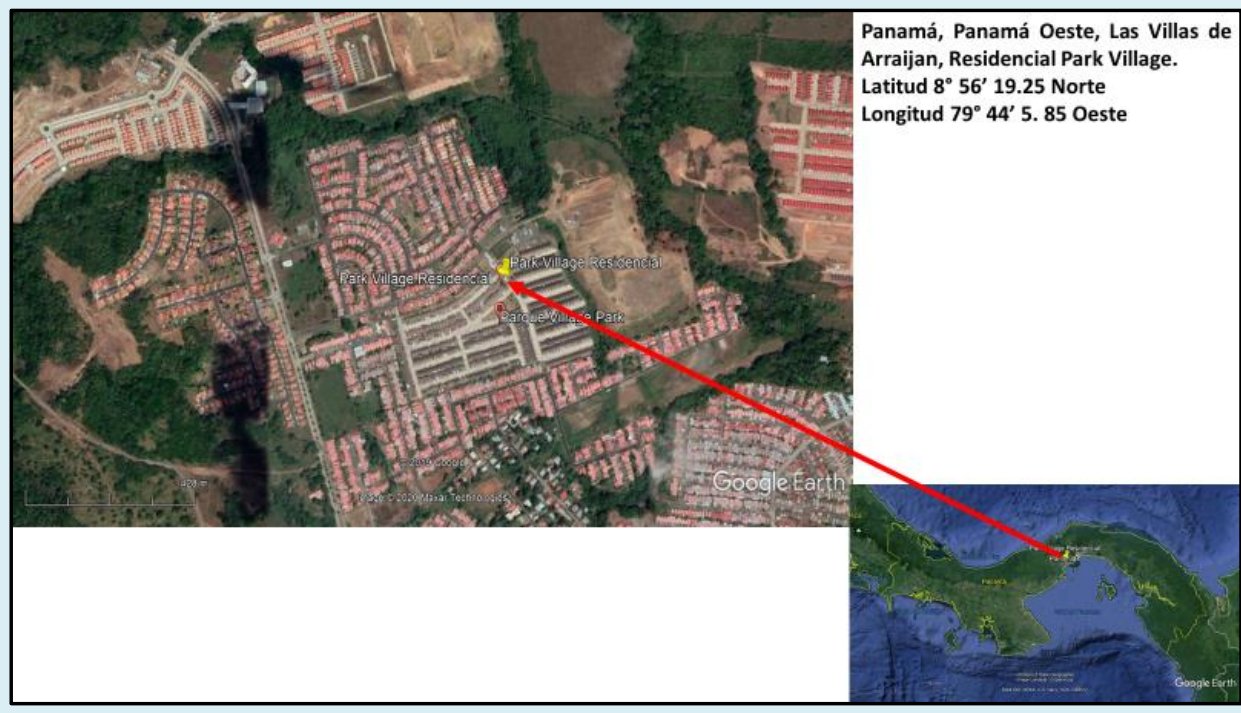

Figura 1. Área de estudio

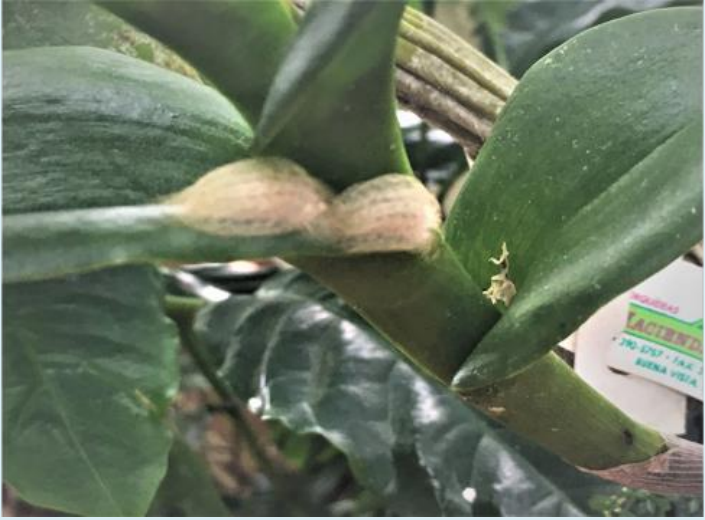

Figura 2. Larvas de H. thaus en hojas de Dendrobium sp.

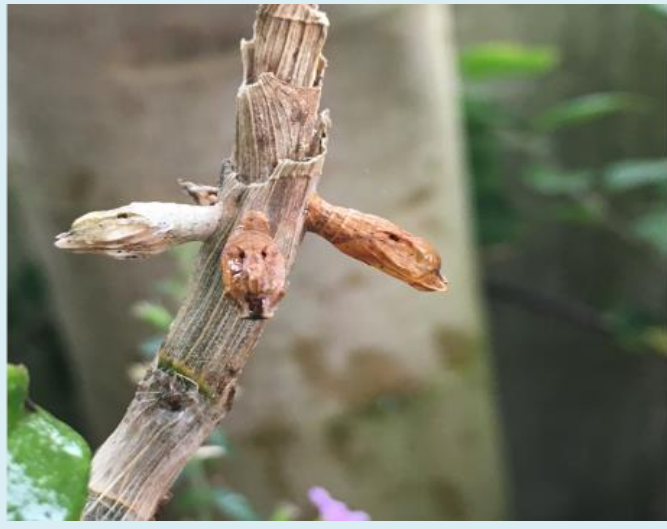

Figura 3. Pupas de $H$. thaus en tallos de Dendrobium sp.

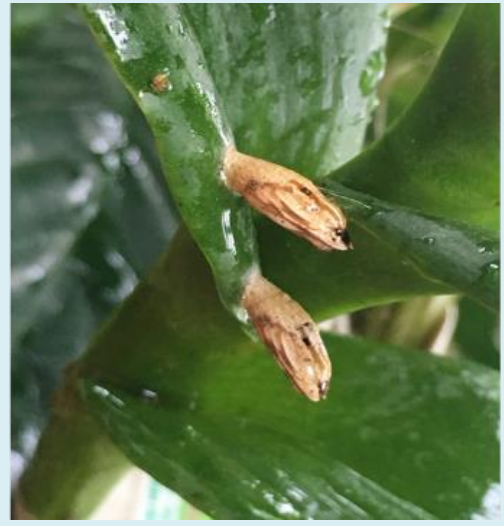

Figura 4. Pupas de H. thaus en hojas de Dendrobium sp.

Transcurrido 17 días, después de pupar la larva, emergió la mosca, la cual corresponde a Lespesia archippivora (Riley) (Diptera: Tachinidae) (Figura 5: A, B, C, D). Las demás pupas (4) emergieron entre los 25 y 30 días los adultos de H. thasus (Figura 6). La época de reproducción de H. thasus se da casi en todos los meses del año, pero es más noble en los meses de julio a septiembre cuando hay más humedad ambiental y las plantas de orquídeas tienen abundantes hojas. También, depende del sector u sitio geográfico donde habiten.

Según Calero (2019), cuando emergen las larvas, ellas se mantienen agrupadas en su plantas hospedera y así se mantienen durante su etapa de vida y desarrollo larval el cual puede durar entre 
25 y 30 días, coincidiendo con la cantidad de días en nuestras observaciones. La época de reproducción se da casi en todos los meses del año dependiendo del sector donde habite.
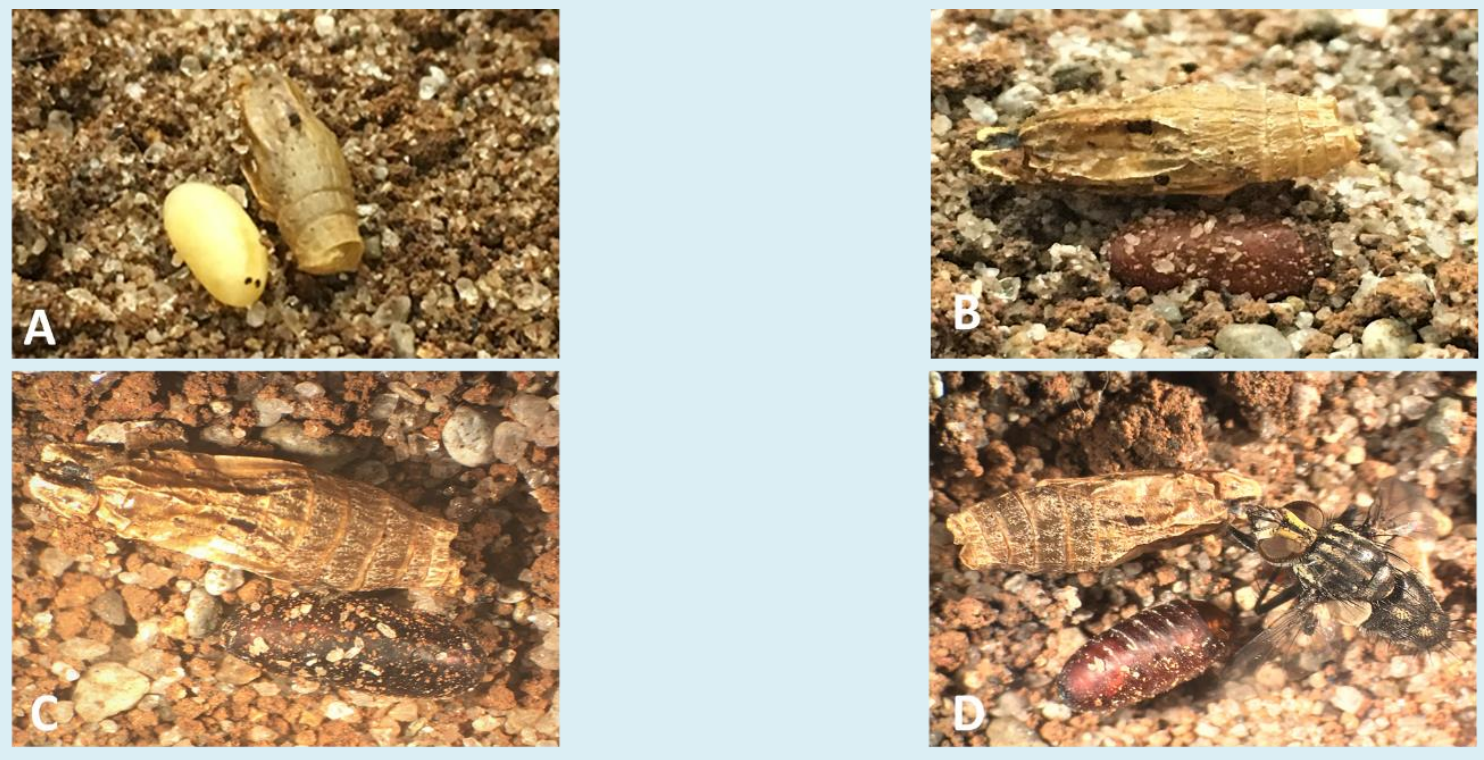

Figura 5. A. Larva de L. archippivora recién pupando. B. Pupa a las 4 horas. C. Pupa a las 5 horas. D. Adulto recién emergido.

Las larvas de H. thasus, se ha reportado utilizando como alimento varias especies de plantas hospederas de la familia Orquideaceae: En Brasil se ha reportado en Brassavola cebolleta y en Oncidium jonesianum (Rech et al., 2009); en Costa Rica en Phaius wallichii (Calero, 2019). H. thasus es una especie de mariposa que no presenta dimorfismo sexual, la coloración en ambos sexos es similar y el tamaño u amplitud alar están entre 13 y 15 mm (medición en 4 adultos: 3 hembras y 1 macho). Esta especie se distribuye desde sur este de México, Costa Rica, Panamá, hasta Brazil y Paraguay. Es una mariposa con hábito diurno, se puede encontrar en diferente hábitat de bosques lluviosos, bordes de bosque, trochas abandonadas, márgenes de quebradas y bosques secundarios.

Las moscas Tachinidae es una de las familias de dípteras más diversas, con aproximadamente 8.500 especies válidas en todo el mundo (O’Hara, 2016). Wood y Zumbado (2010) estiman 130 especies registradas para América Central (incluidas 45 de Costa Rica), pero la fauna real de la región puede llegar a 2.000 especies Zetina Dulce, et al., (2018). Lespesia es un género de Tachinidae, que se encuentra entre los más grandes e importantes del nuevo mundo (Sabrosky, 1980). Este género contiene 66 especies, de las cuales 37 se encuentran en la región Neotropical (Guimarães, 1983; Toma, 2010). Algunas especies han sido reportadas parasitando larvas de varias familias, particularmente Noctuidae, Notodontidae, Saturniidae y Sphingidae (Guimarães 1977, Wood \& Zumbado 2010); no se tenía registro que parasitaba especies de la familia Riodinidae. L. archippivora es un parasitoide presente en Panamá y con distribución en varios países de la región como Estados Unidos, Canadá, México, Hawaii, Puerto Rico, Cuba, Honduras, Nicaragua, Venezuela Chile, Argentina y Brazil. Este es el primer registro de la especie de Tachinidae, Lespesia archippivora parasitando estadios inmaduros de mariposas Riodinidae, H. thasus. 
De acuerdo con Etchegaray \& Toshiyuki (1975) y Beneway (1963), el número de insectos que son parasitados por L. archippivora comprende 25 especies de lepidópteros y un himenóptero, con este reporte, adicional a tres más reportados anteriormente en otros estudios, suman 29 especies de lepidópteros siendo estos: Quadrus cerialis Stoll, 1782 (Lepidoptera: Hesperiidae) (Santos-Murgas, 2019), Halysidota tessellaris (Lepidoptera: Erebidae) (Ábrego \& Santos-Murgas, 2019) y Dione juno (Cramer, 1779) (Lepidoptera: Nymphalidae) (Santos-Murgas, 2020). En las islas hawaianas, las especies de mariposas parasitadas por L. archippivora incluyen: D. plexippus (L.); Pseudoletia unipuncta (Haworth); Pieris rapae (L.); Hedylepta accepta (Butler); Vanessa cardui (L.); Heliothis zea (Boddie); Agrotis ypsilon (Rottemburg); Hedylepta blackburni (Butler) Etchegaray \& Toshiyuki (1975).

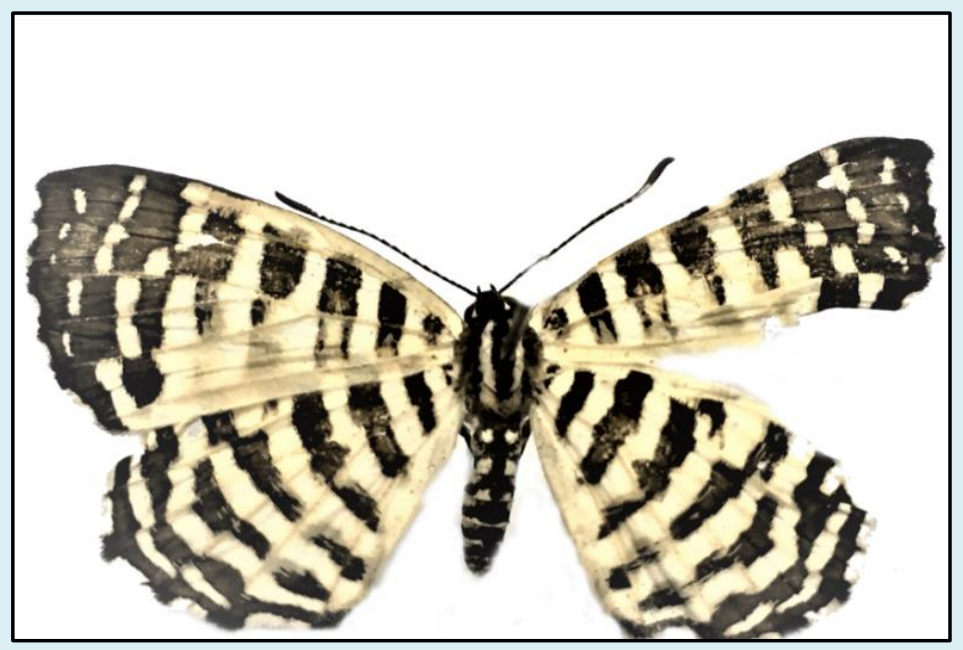

Figura 6. Adulto de Hyphilaria thasus

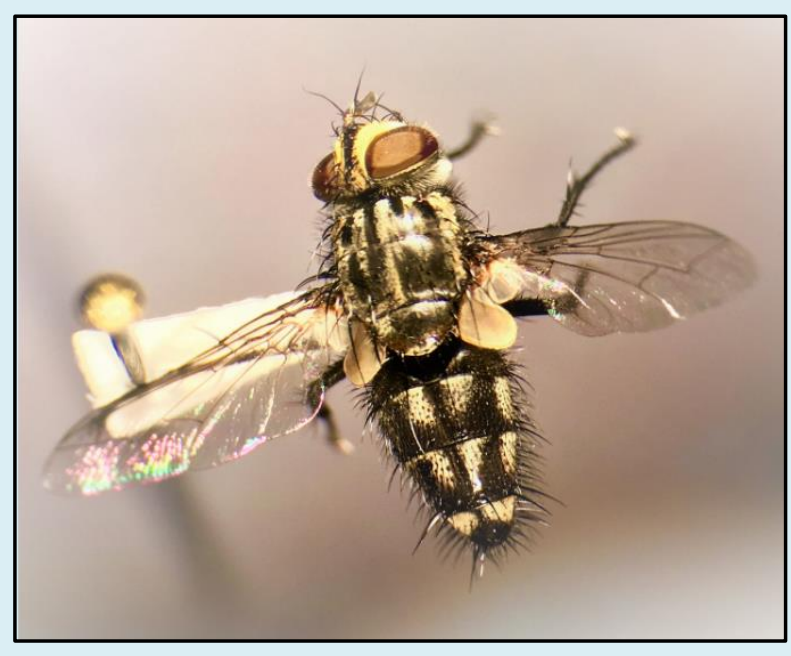

Figura 7. Adulto de Lespesia archippivora

\section{CONFLICTO DE INTERESES}

Los autores presentes declaran que no existe intereses de conflicto.

\section{REFERENCIAS CITADAS}

ABREGO, J., SANTOS-MURGAS, A. 2019. Ocurrencia de multiparasitismo sobre la larva de Halysidota tessellaris (Lepidoptera: Erebidae) en Panamá. Tecnociencia Vol. (21), N². 5-11.

CALERO RICARDO. 2019. Hyphilaria thasus (Stoll, 1780). Área de Conservación de Guanacaste, Fuente de Vida y Desarrollo. $\quad \underline{\text { https://www.acguanacaste.ac.cr/paginas-de-especies/insectos/111-riodinidae/4598-i- }}$ hyphilaria-thasus-i-riodinidae

ETCHEGARAY B. \& NISHIDA, T. 1975. Biology of Lespesia archippivora (Diptera: Tachinidae). Proceedings, Hawaiian Entomological Society Vol. XXII, No. 1, August,41-49.

GARWOOD K. \& LEHMAN R. 2012. Butterflies of Central America, A Photophic Checklist of Common Species; Volume 2: Lycaenidae \& Riodinidae: The Hairstreaks and Metalmarks; Printed in McAllen, Texas, USA.

GUIMARÃES J. 1983. Taxonomy of Brazilian flies of the genus Lespesia Robineau-Desvoidy (Diptera, Tachinidae). Papéis Avulsos de Zoologia 35: 11-30. 
GUIMARÃES J. 1977. Host-parásito y parásito-anfitrión del catálogo de Tachinidae sudamericanos (Diptera). Arquivos Zool. 29: 1-131.

LAMAS, G. 2004. Checklist of Neotropical Lepidoptera, Part 4ạa: Introduction-xvi. ATL + Scient. publ., Gainesville, Fl.

O'HARA J., CERRETTI P. 2016. Annotated catalogue of the Tachinidae (Insecta, Diptera) of the Afrotropical Region, with the description of seven new genera. Canadian Entomologist 130: 751-774. DOI: 10.4039/Ent130751-6.

RECH, A., JARDIN, R. \& JARDIN, R.2009. Primeira ocurrencia de fitofagia de frutos e sementes de Orchideaceae por Hyphilaria thasus Stoll (Lepidoptera, Riodinidae) no Brazil. Revista Brasileña de Horticultura Ornamental, Vol. 14, No. 209-211.

SABROSKY, C. 1980. A revised key to the Nearctic species of Lespesia (Diptera: Tachinidae). Ann. Entomol. Soc. Am. 73: 63-73.

SANTOS-MURGAS, A. 2019. Quadrus cerialis Stoll, 1782 (Lepidoptera: Hesperiidae) y su parasitoide Lespesia archippivora (Diptera: Tachinidae) en Panamá. Revista Nicaragüense de Entomología, №. 162, 1-11.

SANTOS-MURGAS, A., MARTíNEZ, A. \& RODRíGUEZ M. 2020. Enemigos naturales de Dione juno (Cramer, 1779) (Lepidoptera: Nymphalidae) en cultivo de Passiflora edulis sims, J. (1818) Centro Regional Universitario de Coclé, Universidad de Panamá. Tecnociencia Vol. (22), 97-108.

TOMA, R. 2010. Contribuição ao conhecimento das espécies venezuelanas de Lespesia Robineau-Desvoidy (Diptera, Tachinidae, Exoristinae), com descrição de novas espécies. Rev. Brasil. Entomol. 54: 165-172.

WOOD, D. \& ZUMBADO, M. 2010. Tachinidae (tachinid flies, parasitic flies). In: Brown BV, Borkent A, Cumming JM, Wood DM, Woodley N.E., Zumbado M.A. (Eds). Manual of Central American Diptera. Vol. 2. NRC Research Press, Ottawa, 1343-1417.

ZETINA, D., NAPOLES, R., RAMOS C. \& SÁNCHEZ C. 2018. Checklist of Tachinidae (Insecta, Diptera) in México. Transactions American Entomological Societi, TAES 144: 1-89.

Citar como: Santos, A. \& Jaén, L. 2020. Observaciones biológicas de Hyphilaria thasus (Stoll) (Lepidoptera, Riodinidae) y su parasitoide en Panamá. Mesoamericana 24(1): 28-33 